\title{
A Novel Approach towards Bipolar Soft Sets and Their Applications
}

\author{
Tahir Mahmood iD \\ Department of Mathematics and Statistics, International Islamic University Islamabad, Islamabad, Pakistan \\ Correspondence should be addressed to Tahir Mahmood; tahirbakhat@iiu.edu.pk
}

Received 9 August 2020; Revised 14 September 2020; Accepted 24 September 2020; Published 24 October 2020

Academic Editor: Ali Jaballah

Copyright (C) 2020 Tahir Mahmood. This is an open access article distributed under the Creative Commons Attribution License, which permits unrestricted use, distribution, and reproduction in any medium, provided the original work is properly cited.

\begin{abstract}
The notion of bipolar soft sets has already been defined, but in this article, the notion of bipolar soft sets has been redefined, called T-bipolar soft sets. It is shown that the new approach is more close to the concept of bipolarity as compared to the previous ones, and further it is discussed that so far in the study of soft sets and their generalizations, the concept introduced in this manuscript has never been discussed earlier. We have also discussed the operational laws of T-bipolar soft sets and their basic properties. In the end, we have deliberated the algebraic structures associated with T-bipolar soft sets and the applications of T-bipolar soft sets in decision-making problems.
\end{abstract}

\section{Introduction}

To handle the uncertainty has always been a problem for the researchers and decision makers as it appears in almost every field of real life and all sciences including basic sciences, management sciences, social sciences, and information sciences. Many efforts have been made to cope with this concern. The first compact attempt in this direction was made by Zadeh [1] when he familiarized the notion of fuzzy sets in 1965. In 1982, Pawlak [2] familiarized the notion of rough sets. Although these theories have their own advantages and these theories proved their effectiveness, the theory of soft sets by Molodtsov [3] in 1999 did shovel work as it generalizes both the theories. Maji et al. [4] furnished some operations to soft sets. Later on, Ali et al. [5] piercing out some inadequacies in the operations defined in [4] bequeathed some new operations to soft sets like extended union, restricted union, restricted intersection, and the restricted difference of two soft sets. In [6], Ali et al. deliberated some algebraic structures associated with the new defined operations on soft sets. Aktaş and Çağman [7] evidenced that soft sets generalize both fuzzy sets and rough sets and they are pragmatic soft sets in group theory. After the remarkable start of the era of soft sets, many researchers put their share in the progress of the theory of soft sets, for example, Acar et al. [8] presented the notion of soft rings, Sezer and Atagün [9] originated soft vector spaces, Ali et al. [10] represented graphs based on neighbourhoods and soft sets, Shabir and Naz [11] opened the notion of soft topological spaces, Sezer et al. [12] worked on soft intersection semigroups, Ali et al. [13] initiated the notion of lattice ordered soft sets, and Cagman [14] initiated a new approach in soft set theory.

The applications of soft sets in decision making were initiated by Maji et al. [15] in 2002. Since then many other authors contributed in this direction, for example, Cagman and Enginoglu [16, 17] and Kong et al. [18] did copious work in the applications of soft sets in decision making. For more studies and applications of soft sets, one may study [19-23].

The notion of fuzzy soft sets was introduced by Maji et al. [24]. Deng and Wang [25] espoused object parameter methodology for predicting unknown data in incomplete fuzzy soft sets. Naz and Shabir [26] instigated the study of algebraic structures associated with fuzzy soft sets. Roy and Maji [27] toiled on fuzzy soft set theoretic approach to decision-making problems. For more applications of fuzzy soft sets in decision making and other fields, one may study [28-34]. 
The notion of bipolar-valued fuzzy sets was instigated by Lee [35] in 2000. Abdullah et al. [36] commenced the perception of bipolar fuzzy soft sets and applied this perception in a decision-making problem. In 2013, Shabir and $\mathrm{Naz}$ [37] instigated the idea of bipolar soft sets, and then keeping this concept in view, Naz and Shabir [38] familiarized the idea of fuzzy bipolar soft sets and studied their algebraic structures and their applications. In 2014, Karaaslan and Karatas [39] espoused a different methodology to introduce bipolar soft sets, and later on, Karaaslan et al. [40] toiled on bipolar soft groups. For additional work and applications of the impression of bipolarity in soft sets and allied topics, one may study [41-45].

If we sum up all the above debate, then we noticed that keeping in view the association between fuzzy sets and soft sets and keeping in view the significance of bipolar-valued fuzzy sets, two attempts have been made to define bipolar soft sets: one by Shabir and Naz and the other by Karaaslan and Karatas. But if we notice, then we come to know that in both approaches, the conception of bipolar soft sets has some shortcomings, which we will discuss in our upcoming sections of the article (see Remark 1). So keeping this downside of the defined bipolar soft sets, in this article, we have embraced a new approach to define bipolar soft set and we named it T-bipolar soft set. Rest of the article is organized as follows:

(1) In Section 2 of the article, we have given some basic definitions to make the article self-contained and to justify redefining the notion of bipolar soft set.

(2) In Section 3 of the article, the notion of T-bipolar soft sets is familiarized, its basic operational laws are given, and related results are conferred.

(3) In Section 4, some algebraic structures are discussed associated with new defined T-BSSs.

(4) In Section 5, some applications of T-BSSs towards decision making are discussed.

(5) In Section 6, conclusion of the work presented is drawn and some future directions are discussed.

\section{Preliminaries}

In this section of the article, we will provide and deliberate some basic definitions of fuzzy sets, intuitionistic fuzzy sets, bipolar-valued fuzzy sets, soft sets, double framed soft sets, and bipolar-valued soft sets to make the article self-contained and also to justify the need to define T-bipolar soft sets. We will also debate the motivation to define T-bipolar soft sets.

Definition 1 (see [1]). Let $A$ be a nonempty set. Then, a fuzzy set in $A$ is characterized by a membership function $f: A \longrightarrow[0,1]$.

Definition 2 (see [3]). Let $A$ be a nonempty set of parameters and $U$ be an initial universe. Then, a soft set $(F, A)$ over $U$ is characterized by a set valued function $F: A \longrightarrow P(U)$.
Definition 3 (see [46]). Let $A$ be a nonempty set. Then, an intuitionistic fuzzy set in $A$ is characterized by two functions $f: A \longrightarrow[0,1]$ and $g: A \longrightarrow[0,1]$, where $f$ is called a membership function and $g$ is called nonmembership function. The condition that the sum of the values of $f$ and $g$ must belong to $[0,1]$ is the part of the definition of intuitionistic fuzzy set.

Definition 4 (see [47]). Let $A$ be a nonempty set of parameters and $U$ be an initial universe. Then, a double framed soft set over $U$ is characterized by two set valued functions F: $A \longrightarrow P(U)$ and $G: A \longrightarrow P(U)$.

Definition 5 (see [35]). Let $A$ be a nonempty set. Then, a bipolar-valued fuzzy set in $A$ is characterized by two functions $f: A \longrightarrow[0,1]$ and $g: A \longrightarrow[-1,0]$, where for some $x \in A, f(x)$ denotes the satisfaction degree of the element $x$ to the property corresponding to the bipolarvalued fuzzy set, which we denote by $\langle f, g, A\rangle$, and further $g(x)$ denotes the satisfaction degree of $x$ to some implicit counterproperty of the bipolar-valued fuzzy set $\langle f, g, A\rangle$.

Definition 6 (see [37]). Let $A$ be a nonempty set of parameters, $A=\{x: x \in A\}$ denotes the NOT set of $A$, and let $U$ be an initial universe. Then, a bipolar soft set, denoted by $(F, G, A)$, over $U$ is characterized by two set valued functions $F: A \longrightarrow P(U)$ and $G: A \longrightarrow P(U)$ such that for all $x \in A, F(x) \cap G(x)=\varnothing$ (empty set).

Definition 7 (see [39]). Let $A$ be a parameter set and $A_{1}$ and $A_{2}$ be two nonempty subsets of $A$ such that $\left(A_{1} \cup A_{2}=A\right)$ and $A_{1} \cap A_{2}=\varnothing$. Then, the triplet $(F, G, A)$ is thought to be a bipolar soft set over $U$, where $F$ and $G$ are set valued mappings given by $\mathrm{F}: A_{1} \longrightarrow P(U)$ and $\mathrm{G}: A_{2} \longrightarrow P(U)$ such that $F(x) \cap G(f(x))=\varnothing$, where $f: A_{1} \longrightarrow A_{2}$ is a bijective function.

Remark 1. From above definitions, we note that

(1) The definitions of fuzzy sets and that of soft sets have same characteristics in the sense that

(i) Both are characterized by a single function

(ii) Both have a single set as domain set

(iii) Both have a single set, which is a lattice in either case, as codomain set

(2) The definitions of intuitionistic fuzzy sets and that of double framed soft sets have same characteristics in the sense that

(i) Both are characterized by two functions

(ii) Both have a single set as domain set for both the functions

(iii) Both have a single set, which is a lattice in either case, as codomain set for both the functions

(3) But this is not the case for bipolar-valued fuzzy sets as compared to the definitions of bipolar soft sets defined in $[37,39]$ 
All this dialogue demonstrates that the space to define bipolar soft set has not yet been filled. As every definition in mathematics has its own importance and it does not mean that the already existing definitions of bipolar soft sets are of no use and the proposed definition of bipolar soft set will nullify the existing definitions, but the purpose to redefine the notion of bipolar soft set is, one to elaborate the notion of bipolarity in soft sets more affectively and the other is, as there is, up to the best of our knowledge, no such type of situation is discussed in soft sets earlier.

Now, we deliberate some basic definitions connected to soft sets. In this section, from now onwards, $E$ will denote a set of parameters, $A, B, C \ldots \subseteq E$, and $U$ will denote an initial universe. Further, the set of all soft sets over $U$ will be denoted by $(S S)_{(U)}$.

Definition 8 (see [14]). Let $\left(F_{1}, A\right)$ and $\left(F_{2}, B\right) \in(\mathrm{SS})_{(U)}$. Then, $\left(F_{1}, A\right)$ is called a soft subset of $\left(F_{2}, B\right)$ if

(i) $A \subseteq B$

(ii) For all $a \in A, F_{1}(a) \subseteq F_{2}(a)$

Then, we write $\left(F_{1}, A\right) \subseteq\left(F_{2}, B\right) .\left(F_{1}, A\right)$ and $\left(F_{2}, B\right)$ are said to be soft equal if and only if $\left(F_{1}, A\right) \subseteq\left(F_{2}, B\right)$ and $\left(F_{2}, B\right) \subseteq\left(F_{1}, A\right)$. Then, we write $\left(F_{1}, A\right)=\left(F_{2}, B\right)$.

Definition 9 (see [14]). Let $(F, A) \in(\mathrm{SS})_{(U)}$. Then,

(i) Complement of $(F, A)$ is designated and specified by $(F, A)^{c}=\left(F^{c}, A\right)$ where $F^{c}(a)=U-F(a)$, for all $a \in A$

(ii) $(F, A)$ is said to be null if and only if for all $a \in A, F(a)=\varnothing$

(iii) $(F, A)$ is said to be absolute if and only if for all $a \in A, F(a)=U$

Definition 10 (see [4]). Let $\left(F_{1}, A\right)$ and $\left(F_{2}, B\right) \in(\mathrm{SS})_{(U)}$. Then,

(i) “AND" product of $\left(F_{1}, A\right)$ and $\left(F_{2}, B\right)$ is designated and demarcated by $\left(F_{1}, A\right) \wedge\left(F_{2}, B\right)=\left(F_{3}, A \times B\right)$ where $F_{3}(a, b)=F_{1}(a) \cap F_{2}(b)$ for all $(a, b) \in A \times B$

(ii) "OR" product of $\left(F_{1}, A\right)$ and $\left(F_{2}, B\right)$ is designated and demarcated by $\left(F_{1}, A\right) \vee\left(F_{2}, B\right)=\left(F_{3}, A \times B\right)$ where $\quad F_{3}(a, b)=F_{1}(a) \cup F_{2}(b)$ for all $(a, b) \in A \times B$

Definition 11 (see [4]). Let $\left(F_{1}, A\right)$ and $\left(F_{2}, B\right) \in(\mathrm{SS})_{(U)}$. Then, "union" (which we may also call extended union) of $\left(F_{1}, A\right)$ and $\left(F_{2}, B\right)$ is designated and demarcated by $\left(F_{1}, A\right) \cup_{E}\left(F_{2}, B\right)=(H, A \cup B)$, where

$$
H(e)= \begin{cases}F_{1}(e), & \text { if } e \in A-B, \\ F_{2}(e), & \text { if } e \in B-A, \\ F_{1}(e) \cup F_{2}(e), & \text { if } e \in A \cap B .\end{cases}
$$

Definition 12 (see [5]). Let $\left(F_{1}, A\right)$ and $\left(\left(F_{2}, B\right) \in(\mathrm{SS})_{(U)}\right)$. Then, "extended intersection" of $\left(F_{1}, A\right)$ and $\left(F_{2}, B\right)$ is designated and demarcated by $\left(F_{1}, A\right) \cap_{E}\left(F_{2}, B\right)=(H, A \cup B)$, where

$$
H(e)= \begin{cases}F_{1}(e), & \text { if } e \in A-B, \\ F_{2}(e), & \text { if } e \in B-A, \\ F_{1}(e) \cap F_{2}(e), & \text { if } e \in A \cap B .\end{cases}
$$

Definition 13 (see [5]). Let $\left(F_{1}, A\right)$ and $\left(F_{2}, B\right) \in(\mathrm{SS})_{(U)}$ such that $(A \cap B)$ is nonempty. Then,

(i) "Restricted union" of $\left(F_{1}, A\right)$ and $\left(F_{2}, B\right)$ is designated and demarcated by $\left(F_{1}, A\right) \cup_{R}\left(F_{2}, B\right)=$ $(H, A \cap B)$, where $H(e)=F_{1}(e) \cup F_{2}(e)$

(ii) "Restricted intersection" of $\left(F_{1}, A\right)$ and $\left(F_{2}, B\right)$ is designated and demarcated by $\left(F_{1}, A\right) \cap_{R}\left(F_{2}, B\right)=$ $(H, A \cap B)$, where $H(e)=F_{1}(e) \cap F_{2}(e)$

\section{T-Bipolar Soft Set}

In this section, we will familiarize the perception of T-bipolar soft set (T-BSS), we will delineate binary operations for T-BSSs, and we will also deliberate some basic properties and some results concomitant with these concepts. First, we contemplate the succeeding example.

Example 1. Let us consider the case, where a researcher Dr. Shabir wants to submit his four research articles $a_{1}$ (on homological algebra), $a_{2}$ (on fuzzy sets), $a_{3}$ (on soft sets), and $a_{4}$ (on rough sets) in some research journals. For the purpose, he has to propose some potential referees and also he has the option to oppose some referees. Keeping in view all the aspects, he prepared a set $X=\left\{x_{1}, x_{2}, x_{3}, x_{4}, x_{5}, x_{6}\right\}$ of some proposed referees as well as a set $Y=\left\{y_{1}, y_{2}, y_{3}, y_{4}, y_{5}\right\}$ of some referees to oppose. Hence, in this case, he has under consideration the set $U=\left\{x_{1}, x_{2}, x_{3}, x_{4}, x_{5}, x_{6}, y_{1}, y_{2}, y_{3}, y_{4}, y_{5}\right\}$ of all referees. For each of his article, he selects some referees from $X$ to propose and selects some referees from $Y$ to oppose. Keeping in view all the aspects for the article

(i) $a_{1}$ (on homological algebra), he decided to propose $x_{2}, x_{3}, x_{4}$ and he opposed $y_{1}, y_{2}$

(ii) $a_{2}$ (on fuzzy sets), he decided to propose $x_{1}, x_{3}, x_{6}$ and he opposed $y_{2}, y_{4}$

(iii) $a_{3}$ (on soft sets), he decided to propose $x_{4}, x_{5}$ and he opposed $y_{3}$

(iv) $a_{4}$ (on rough sets), he decided to propose $x_{1}, x_{5}, x_{6}$ and he opposed $y_{1}, y_{4}, y_{5}$

Note that all this information can be modeled mathematically as follows: let $A=\left\{a_{1}, a_{2}, a_{3}, a_{4}\right\}$, $X=\left\{x_{1}, x_{2}, x_{3}, x_{4}, x_{5}, x_{6}\right\}$, and $Y=\left\{y_{1}, y_{2}, y_{3}, y_{4}, y_{5}\right\}$. Define $(F: A \longrightarrow P(X))$ as $F\left(a_{1}\right)=\left\{x_{2}, x_{3}, x_{4}\right\}, F\left(a_{2}\right)$ $=\left\{x_{1}, x_{3}, x_{6}\right\}, F\left(a_{3}\right)=\left\{x_{4}, x_{5}\right\}, F\left(a_{4}\right)=\left\{x_{1}, x_{5}, x_{6}\right\}$, and $(G: A \longrightarrow P(Y)) \quad$ as $\quad G\left(a_{1}\right)=\left\{y_{1}, y_{2}\right\}, G\left(a_{2}\right)=$ $\left\{y_{2}, y_{4}\right\}, G\left(a_{3}\right)=\left\{y_{3}\right\}, G\left(a_{4}\right)=\left\{y_{1}, y_{4}, y_{5}\right\}$. 
Here, it can be perceived that both the functions $F$ and $G$ have common domain $A$, and codomains of $F$ and $G$ have nothing in common except the empty set $\phi$. Further we notice that same is the case in bipolar-valued fuzzy sets. Hence, we have the following definition.

Definition 14. Let $E$ be a set of parameters, $A \subseteq E$, and $U$ be an initial universe, $X \subset U$ and $Y=U-X$. Then, a triplet $F, G, A$ is said to be a T-BSS over $U$, where $F$ and $G$ are set valued mappings given by $F: A \longrightarrow P(X)$ and $\mathrm{G}: A \longrightarrow P(Y)$. In this case, we write $(F, G, A)=$ $\{\langle a, F(a), G(a): F(a) \in P(X)$ and $G(a) \in P(Y)\rangle\}$ or simply $(F, G, A)=\{\langle a, F(a), G(a)\rangle\}$. The collection of all T-BSSs over $U$ is denoted by $(T-B S S)_{(U)}$.

Remark 2. Let $A=\left\{a_{1}, a_{2}, a_{3}, \ldots, a_{l}\right\} \subseteq E, X=\left\{x_{1}, x_{2}\right.$, $\left.x_{3}, \ldots, x_{m}\right\}, Y=\left\{y_{1}, y_{2}, y_{3}, \ldots, y_{n}\right\}$, and $(F, G, A)$ be corresponding T-BSS. Then, we can represent $(F, G, A)$ as follows (Table 1).

$$
\begin{aligned}
& \zeta_{i j k}=\left(\mu_{j}, v_{k}\right)= \begin{cases}(0,0) & \text { if } x_{j} \notin F\left(a_{i}\right) \text { and } y_{k} \notin F\left(a_{i}\right), \\
(1,0) & \text { if } x_{j} \in F\left(a_{i}\right) \text { and } y_{k} \notin F\left(a_{i}\right), \\
(0,1) & \text { if } x_{j} \notin F\left(a_{i}\right) \text { and } y_{k} \in F\left(a_{i}\right), \\
(1,1) & \text { if } x_{j} \in F\left(a_{i}\right) \text { and } y_{k} \in F\left(a_{i}\right),\end{cases} \\
& \zeta_{i j k}^{*}=\mu_{j}, \\
& \zeta_{i j k}^{\circ}=v_{k} .
\end{aligned}
$$

Example 2. A university wants to appoint a permanent faculty member from the set $A=\left\{a_{1}, a_{2}, a_{3}, a_{4}, a_{5}\right\}$ of visiting faculty members. For the purpose, the university authorities constitute two panels $X=\left\{x_{1}, x_{2}, x_{3}, x_{4}\right\}$ and $Y=\left\{y_{1}, y_{2}, y_{3}\right\}$ of experts, where panel $X$ consists of members from outside the university and panel $Y$ consists of members from inside the university. Further each member of the panel $X$ will decide about each candidate by considering his/her experience, number of research publications, number of conferences attended, etc., while each member of the panel $Y$ will decide about each candidate by considering his/her regularity and punctuality, attitude towards other faculty members, and behavior with students during class. Now the university authorities decided the selection criteria that towards each candidate each member of the panel $X$ will have to select a candidate by keeping in view his/her positive points while each member of the panel $Y$ has to reject a candidate by keeping in view his/her negative points. According to the decisions taken by the members of the panels $X$, the experts $x_{1}$ and $x_{3}$ are in favor to select the candidate $a_{1}$ while $x_{2}$ and $x_{4}$ decided to remain neutral for the candidate $a_{1}$. Similarly the decisions taken by the members of the panels $Y$, the member $y_{3}$ is not in favor to select the candidate $a_{1}$ while $y_{1}$ and $y_{2}$ decided to remain neutral for the candidate $a_{1}$. Hence, for the candidate $a_{1}$, the situation can be modeled as $\left\langle a_{1},\left\{x_{1}, x_{3}\right\},\left\{y_{3}\right\}\right\rangle$. Now keeping under consideration the decisions taken by all the members from the panels $X$ and $Y$, the result can be modeled mathematically as given in the following T-BSS: $((F, G, A)=$ $\left\{\left\langle a_{1},\left\{x_{1}, x_{3}\right\},\left\{y_{3}\right\}\right\rangle,\left\langle a_{2},\left\{x_{1}, x_{3}, x_{4}\right\},\left\{y_{1}\right\}\right\rangle,\left\langle a_{3},\left\{x_{1}, x_{4}\right\}\right.\right.$, $\left.\left.\left.\left\{y_{1}, y_{3}\right\}\right\rangle,\left\langle a_{4},\left\{x_{2}, x_{3}, x_{4}\right\},\left\{y_{2}, y_{3}\right\}\right\rangle,\left\langle a_{5},\left\{x_{2}, x_{4}\right\},\left\{y_{2}\right\}\right\rangle\right\}\right)$.

Tabular form of the $(F, G, A)$ is given as follows (Table 2).

Definition 15. Let $\left(F_{1}, G_{1}, A\right) \in(T-B S S)_{(U)}$. Then, $\left(F_{1}, G_{1}, A\right)$ is said to be T-bipolar soft subset of $\left(F_{2}, G_{2}, B\right)$ if

(i) $A \subseteq B$

(ii) For all $a \in A, F_{1}(a) \subseteq F_{2}(a)$ and $G_{2}(a) \subseteq G_{1}(a)$.

Then, we write $\left(F_{1}, G_{1}, A\right) \subseteq\left(F_{2}, G_{2}, B\right) .\left(F_{1}, G_{1}, A\right)$ and $\left(F_{2}, G_{2}, B\right)$ are said to be equal if and only if $\left(F_{1}, G_{1}, A\right) \subseteq\left(F_{2}, G_{2}, B\right)$ and $\left(F_{2}, G_{2}, B\right) \subseteq\left(F_{1}, G_{1}, A\right)$. Then, we write $\left(F_{1}, G_{1}, A\right)=\left(F_{2}, G_{2}, B\right)$.

Definition 16. Let $(F, G, A) \in(\mathrm{T}-\mathrm{BSS})_{(U)}$. Then,

(i) Complement of $(F, G, A)$ is denoted and given by $(F, G, A)^{c}=\left(F^{c}, G^{c}, A\right)=\left\{\left\langle a, F^{c}(a)=X-F(a), \quad G^{c}\right.\right.$ $(a)=Y-G(a)\rangle\}$.

(ii) $(F, G, A)$ is said to be null if and only if for all $a \in A, F(a)=\varnothing$ and $G(a)=Y$. In our study, it will further be designated by $\phi$, that is, $\phi=\{\langle a, \varnothing, Y\rangle\}$.

(iii) $(F, G, A)$ is said to be absolute if and only if for all $a \in A, F(a)=X$ and $\mathrm{G}(a)=\varnothing$. In our study, it will further be designated by $\mathscr{A}$ that is, $\mathscr{A}=\{\langle a, X, \varnothing\rangle\}$.

Definition 17. Let $\left(F_{1}, G_{1}, A\right),\left(F_{2}, G_{2}, B\right) \in(\mathrm{T}-\mathrm{BSS})_{(U)}$. Then,

(i) "AND" product of $\left(F_{1}, G_{1}, A\right)$ and $\left(F_{2}, G_{2}, B\right)$ is designated and demarcated by

$$
\begin{aligned}
\left(F_{1}, G_{1}, A\right) \wedge\left(F_{2}, G_{2}, B\right) & =\left\{<(a, b), F_{1}(a) \cap F_{2}(b), G_{1}(a)\right. \\
\cup G_{2}(b)>: & (a, b) \in A \times B\} .
\end{aligned}
$$

(ii) "OR" product of $\left(F_{1}, G_{1}, A\right)$ and $\left(F_{2}, G_{2}, B\right)$ is designated and demarcated by

$$
\begin{aligned}
\left(F_{1}, G_{1}, A\right) & \vee\left(F_{2}, G_{2}, B\right)=\left\{<(a, b), F_{1}(a) \cup F_{2}(b), G_{1}(a)\right. \\
\cap G_{2}(b)>: & (a, b) \in A \times B\} .
\end{aligned}
$$

Proposition 1. Let $\left(F_{1}, G_{1}, A\right), \quad\left(F_{2}, G_{2}, B\right) \quad\left(F_{2}, G_{2}, B\right) \in$ $(T-B S S)_{(U)}$. Then,

(i) $\left[\left(F_{1}, G_{1}, A\right) \wedge\left(F_{2}, G_{2}, B\right)\right]^{c}=\left[\left(F_{1}, G_{1}, A\right)\right]^{c} \vee\left[\left(F_{2}\right.\right.$, $\left.\left.G_{2}, B\right)\right]^{c}$ 
TABLe 1: Tabular form of a T-bipolar soft set.

\begin{tabular}{lccccccccccccc}
\hline$(F, G, A)$ & $\left(x_{1}, y_{1}\right)$ & $\left(x_{1}, y_{2}\right)$ & $\ldots$ & $\left(x_{1}, y_{n}\right)$ & $\left(x_{2}, y_{1}\right)$ & $\left(x_{2}, y_{2}\right)$ & $\ldots$ & $\left(x_{2}, y_{n}\right)$ & $\ldots$ & $\left(x_{m}, y_{1}\right)$ & $\left(x_{m}, y_{2}\right)$ & $\ldots$ & $\left(x_{m}, y_{n}\right)$ \\
\hline$a_{1}$ & $\zeta_{111}$ & $\zeta_{112}$ & $\ldots$ & $\zeta_{11 n}$ & $\zeta_{121}$ & $\zeta_{122}$ & $\ldots$ & $\zeta_{12 n}$ & $\ldots$ & $\zeta_{1 m 1}$ & $\zeta_{1 m 2}$ & $\ldots$ & $\zeta_{1 m n}$ \\
$a_{2}$ & $\zeta_{211}$ & $\zeta_{212}$ & $\ldots$ & $\zeta_{21 n}$ & $\zeta_{221}$ & $\zeta_{222}$ & $\ldots$ & $\zeta_{22 n}$ & $\ldots$ & $\zeta_{2 m 1}$ & $\zeta_{2 m 2}$ & $\ldots$ & $\zeta_{2 m n}$ \\
$a_{3}$ & $\zeta_{311}$ & $\zeta_{312}$ & $\ldots$ & $\zeta_{31 n}$ & $\zeta_{321}$ & $\zeta_{322}$ & $\ldots$ & $\zeta_{32 n}$ & $\ldots$ & $\zeta_{3 m 1}$ & $\zeta_{3 m 2}$ & $\ldots$ & $\zeta_{3 m n}$ \\
$\ldots$ & $\ldots$ & $\ldots$ & $\ldots$ & $\ldots$ & $\ldots$ & $\ldots$ & $\ldots$ & $\ldots$ & $\ldots$ & $\ldots$ & $\ldots$ & $\ldots$ & $\ldots$ \\
$\ldots$ & $\ldots$ & $\ldots$ & $\ldots$ & $\ldots$ & $\ldots$ & $\ldots$ & $\ldots$ & $\ldots$ & $\ldots$ & $\ldots$ & $\ldots$ & $\ldots$ & $\ldots$ \\
$a_{l}$ & $\zeta_{l 11}$ & $\zeta_{112}$ & $\ldots$ & $\zeta_{l 1 n}$ & $\zeta_{l 21}$ & $\zeta_{122}$ & $\ldots$ & $\zeta_{l 2 n}$ & $\ldots$ & $\zeta_{l m 1}$ & $\zeta_{l m 2}$ & $\ldots$ & $\zeta_{l m n}$ \\
\hline
\end{tabular}

TAble 2: Tabular form of the T-bipolar soft set $(F, G, A)$.

\begin{tabular}{lcccccccccccc}
\hline$(F, G, A)$ & $\left(x_{1}, y_{1}\right)$ & $\left(x_{1}, y_{2}\right)$ & $\left(x_{1}, y_{3}\right)$ & $\left(x_{2}, y_{1}\right)$ & $\left(x_{2}, y_{2}\right)$ & $\left(x_{2}, y_{3}\right)$ & $\left(x_{3}, y_{1}\right)$ & $\left(x_{3}, y_{2}\right)$ & $\left(x_{3}, y_{3}\right)$ & $\left(x_{4}, y_{1}\right)$ & $\left(x_{4}, y_{2}\right)$ & $\left(x_{4}, y_{3}\right)$ \\
\hline$a_{1}$ & $(1,0)$ & $(1,0)$ & $(1,1)$ & $(0,0)$ & $(0,0)$ & $(0,1)$ & $(1,0)$ & $(1,0)$ & $(1,1)$ & $(0,0)$ & $(0,0)$ & $(0,1)$ \\
$a_{2}$ & $(1,1)$ & $(1,0)$ & $(1,0)$ & $(0,1)$ & $(0,0)$ & $(0,0)$ & $(1,1)$ & $(1,0)$ & $(1,0)$ & $(1,1)$ & $(1,0)$ & $(1,0)$ \\
$a_{3}$ & $(1,1)$ & $(1,0)$ & $(1,1)$ & $(0,1)$ & $(0,0)$ & $(0,1)$ & $(0,1)$ & $(0,0)$ & $(0,1)$ & $(1,1)$ & $(1,0)$ & $(1,1)$ \\
$a_{4}$ & $(0,0)$ & $(0,1)$ & $(0,1)$ & $(1,0)$ & $(1,1)$ & $(1,1)$ & $(1,0)$ & $(1,1)$ & $(1,1)$ & $(1,0)$ & $(1,1)$ & $(1,1)$ \\
$a_{5}$ & $(0,0)$ & $(0,1)$ & $(0,0)$ & $(1,0)$ & $(1,1)$ & $(1,0)$ & $(0,0)$ & $(0,1)$ & $(0,0)$ & $(1,0)$ & $(1,1)$ & $(1,0)$ \\
\hline
\end{tabular}

(ii) $\left[\left(F_{1}, G_{1}, A\right) \vee\left(F_{2}, G_{2}, B\right)\right]^{c}=\left[\left(F_{1}, G_{1}, A\right)\right]^{c} \wedge$ $\left[\left(F_{2}, G_{2}, B\right)\right]^{c}$

Proof

(i) $\left[\left(F_{1}, G_{1}, A\right) \wedge\left(F_{2}, G_{2}, B\right)\right]^{c}=\left\{\left\langle(a, b), F_{1}(a) \cap F_{2}(b)\right.\right.$, $\left.\left.G_{1}(a) \cup G_{2}(b)\right\rangle\right\}^{c}=\left\{\left\langle(a, b), X-\left(F_{1}(a) \cap F_{2}(b)\right), \quad Y-\right.\right.$ $\left.\left.\left(G_{1}(a) \cup G_{2}(b)\right)\right\rangle\right\}=\left\{\left\langle(a, b),\left(X-F_{1}(a)\right) \cup \quad\left(X-F_{2}\right.\right.\right.$ (b)), $\left.\left.\left(Y-G_{1}(a)\right) \cap\left(Y-G_{2}(b)\right)\right\rangle\right\}=\left\{\left\langle<(a, b), \quad F_{1}^{c}\right.\right.$ $\left.\left.(a) \cup F_{2}^{c}(b), G_{1}^{c}(a) \cap G_{2}^{c}(b)\right\rangle\right\}=\left[\left(F_{1}, G_{1}, A\right)\right]^{c} \vee\left[\left(F_{2}\right.\right.$, $\left.\left.G_{2}, B\right)\right]^{c}$.

(ii) Similar to part (i).

Definition 18. Let $\left(F_{1}, G_{1}, A\right),\left(F_{2}, G_{2}, B\right) \in(T-B S S)_{(U)}$. Then, "extended union" of $\left(F_{1}, G_{1}, A\right)$ and $\left(F_{2}, G_{2}, B\right)$ is designated and demarcated by $\left(\left(F_{1}, G_{1}, A\right) \cup_{E}\left(F_{2}, G_{2}, B\right)=(H, K, A \cup B)\right)$, where

$$
\begin{gathered}
H(e)= \begin{cases}F_{1}(e), & \text { if } e \in A-B, \\
F_{2}(e), & \text { if } e \in B-A, \\
F_{1}(e) \cup F_{2}(e), & \text { if } e \in A \cap B,\end{cases} \\
K(e)= \begin{cases}G_{1}(e), & \text { if } e \in A-B, \\
G_{2}(e), & \text { if } e \in B-A, \\
G_{1}(e) \cap G_{2}(e), & \text { if } e \in A \cap B .\end{cases}
\end{gathered}
$$

Definition 19. Let $\left(F_{1}, G_{1}, A\right), \quad\left(F_{2}, G_{2}, B\right) \in(\mathrm{T}-\mathrm{BSS})_{(U)}$ Then, "extended intersection" of $\left(F_{1}, G_{1}, A\right)$ and $\left(F_{2}, G_{2}, B\right)$ is designated and demarcated by

$$
\left(F_{1}, G_{1}, A\right) \cap_{E}\left(F_{2}, G_{2}, B\right)=(H, K, A \cup B),
$$

where

$$
\begin{gathered}
H(e)= \begin{cases}F_{1}(e), & \text { if } e \in A-B, \\
F_{2}(e), & \text { if } e \in B-A, \\
F_{1}(e) \cap F_{2}(e), & \text { if } e \in A \cap B,\end{cases} \\
K(e)= \begin{cases}G_{1}(e), & \text { if } e \in A-B, \\
G_{2}(e), & \text { if } e \in B-A, \\
G_{1}(e) \cup G_{2}(e), & \text { if } e \in A \cap B .\end{cases}
\end{gathered}
$$

Proposition 2. For any T-BSSs $\left(F_{1}, G_{1}, A\right),\left(F_{2}, G_{2}, B\right)$, and $\left(F_{3}, G_{3}, C\right)$,

(i) $\left(F_{1}, G_{1}, A\right) \cap_{E} \phi=\phi,\left(F_{1}, G_{1}, A\right) \cup_{E} \phi=\left(F_{1}, G_{1}, \quad A\right)$, $\left(F_{1}, G_{1}, A\right) \cap_{E} \mathscr{A}=\left(F_{1}, G_{1}, A\right),\left(F_{1}, G_{1}, A\right) \cup_{E} \mathscr{A}=\mathscr{A}$

(ii) $\left(F_{1}, G_{1}, A\right) \cap_{E}\left(F_{1}, G_{1}, A\right)=\left(F_{1}, G_{1}, A\right),\left(F_{1}, G_{1}, A\right)$ $\cup_{E}\left(F_{1}, G_{1}, A\right)=\left(F_{1}, G_{1}, A\right)$

(iii) $\left(F_{1}, G_{1}, A\right) \cap_{E}\left(F_{2}, G_{2}, B\right)=\left(F_{2}, G_{2}, B\right) \cap_{E}\left(F_{1}, G_{1}\right.$, $A),\left(F_{1}, G_{1}, A\right) \cup_{E}\left(F_{2}, G_{2}, B\right)=\left(F_{2}, G_{2}, B\right) \cup \cup_{E}\left(F_{1}\right.$, $\left.G_{1}, A\right)$

(iv) $\left(F_{1}, G_{1}, A\right) \cup_{E}\left[\left(F_{2}, G_{2}, B\right) \cup_{E}\left(F_{3}, G_{3}, C\right)\right]=\left[\left(F_{1}\right.\right.$, $\left.\left.G_{1}, A\right) \cup_{E}\left(F_{2}, G_{2}, B\right)\right] \cup_{E}\left(F_{3}, G_{3}, C\right)$

(v) $\left(F_{1}, G_{1}, A\right) \cap_{E}\left[\left(F_{2}, G_{2}, B\right) \cup_{E}\left(F_{1}, G_{1}, A\right)\right]=\left(F_{1}, G_{1}\right.$, $A),\left(F_{1}, G_{1}, A\right) \cup_{E}\left[\left(F_{2}, G_{2}, B\right) \cap_{E}\left(F_{1}, G_{1}, A\right)\right]=\left(F_{1}\right.$, $\left.G_{1}, A\right)$

(vi) $\left[\left(F_{1}, G_{1}, A\right)^{c}\right]^{c}=\left(F_{1}, G_{1}, A\right),\left(F_{1}, G_{1}, A\right) \cap_{E}\left[\left(F_{1}, G_{1}\right.\right.$, $A)]^{c}=\phi,\left(F_{1}, G_{1}, A\right) \cup \cup_{E}\left[\left(F_{1}, G_{1}, A\right)\right]^{c}=\mathscr{A}$

(vii) $\left[\left(F_{1}, G_{1}, A\right) \cap_{E}\left(F_{2}, G_{2}, B\right)\right]^{c}=\left[\left(F_{1}, G_{1}, A\right)\right]^{c} \cup_{E}\left[\left(F_{2}\right.\right.$, $\left.\left.G_{2}, B\right)\right]^{c},\left[\left(F_{1}, G_{1}, A\right) \cup \cup_{E}\left(F_{2}, G_{2}, B\right)\right]^{c}=\left[\left(F_{1}, G_{1}, A\right)\right]^{c}$ $\cap_{E}\left[\left(F_{2}, G_{2}, B\right)\right]^{c}$

Proof. We prove (iv) and (vii); rest are straightforward.

(iv) When $x \in A, x \notin B$, and $x \in C$, then 


$$
\begin{aligned}
\left(F_{1}, G_{1}, A\right) \cap \cap_{E}\left[\left(F_{2}, G_{2}, B\right) \cap \cap_{E}\left(F_{3}, G_{3}, C\right)\right] \\
\quad=\left(F_{1}, G_{1}, A\right) \cap_{E}\left(F_{3}, G_{3}, C\right) \\
\quad=\left\{\left\langle\left\{<x \in A \cap C: F_{1}(x) \cap F_{3}(x), G_{1}(x) \cup G_{3}(x)>\right\}\right\rangle\right\}, \\
\cdot\left[\left(F_{1}, G_{1}, A\right) \cap_{E}\left(F_{2}, G_{2}, B\right)\right] \cap_{E}\left(F_{3}, G_{3}, C\right) \\
\quad=\left(F_{1}, G_{1}, A\right) \cap_{E}\left(F_{3}, G_{3}, C\right) \\
\quad=\left\{\left\langle x \in A \cap C: F_{1}(x) \cap F_{3}(x), G_{1}(x) \cup G_{3}(x)\right\rangle\right\} .
\end{aligned}
$$

When $x \in A, x \in B$, and $x \notin C$, then

$$
\begin{aligned}
\left(F_{1}, G_{1}, A\right) \cap \cap_{E}\left[\left(F_{2}, G_{2}, B\right) \cap \cap_{E}\left(F_{3}, G_{3}, C\right)\right] \\
\quad=\left(F_{1}, G_{1}, A\right) \cap_{E}\left(F_{2}, G_{2}, B\right) \\
\quad=\left\{\left\langle x \in A \cap B: F_{1}(x) \cap F_{2}(x), G_{1}(x) \cup G_{2}(x)\right\rangle\right\}, \\
\cdot\left[\left(F_{1}, G_{1}, A\right) \cap_{E}\left(F_{2}, G_{2}, B\right)\right] \cap \cap_{E}\left(F_{3}, G_{3}, C\right) \\
\quad=\left(F_{1}, G_{1}, A\right) \cap_{E}\left(F_{2}, G_{2}, B\right) \\
\quad=\left\{\left\langle x \in A \cap B: F_{1}(x) \cap F_{2}(x), G_{1}(x) \cup G_{2}(x)\right\rangle\right\} .
\end{aligned}
$$

(10)

When $x \notin A, x \in B$, and $x \in C$, then

$$
\begin{aligned}
\left(F_{1}, G_{1}, A\right) \cap_{E}\left[\left(F_{2}, G_{2}, B\right) \cap_{E}\left(F_{3}, G_{3}, C\right)\right] \\
\quad=\left(F_{2}, G_{2}, B\right) \cap_{E}\left(F_{3}, G_{3}, C\right) \\
\quad=\left\{\left\langle x \in B \cap C: F_{2}(x) \cap F_{3}(x), G_{2}(x) \cup G_{3}(x)\right\rangle\right\} \\
\cdot\left[\left(F_{1}, G_{1}, A\right) \cap_{E}\left(F_{2}, G_{2}, B\right)\right] \cap_{E}\left(F_{3}, G_{3}, C\right) \\
\quad=\left(F_{2}, G_{2}, B\right) \cap_{E}\left(F_{3}, G_{3}, C\right) \\
\quad=\left\{\left\langle x \in B \cap C: F_{2}(x) \cap F_{3}(x), G_{2}(x) \cup G_{3}(x)\right\rangle\right\} .
\end{aligned}
$$

When $x \notin A, x \in B$, and $x \in C$, then result is obvious. Hence, it concludes that $\left(F_{1}, G_{1}, A\right) \cap_{E}$ $\left[\left(F_{2}, G_{2}, B\right) \cap_{E} \quad\left(F_{3}, G_{3}, C\right)\right]=\left[\left(F_{1}, G_{1}, A\right) \cap_{E} \quad\left(F_{2}\right.\right.$, $\left.\left.G_{2}, B\right)\right] \cap_{E}\left(F_{3}, G_{3}, C\right)$. Similarly $\left(F_{1}, G_{1}, A\right) \cup_{E}$ $\left[\left(F_{2}, G_{2}, B\right) \cup_{E}\left(F_{3}, G_{3}, C\right)\right]=\left[\left(F_{1}, G_{1}, A\right) \cup_{E}\left(F_{2}, G_{2}\right.\right.$, $B)] \cup_{E}\left(F_{3}, G_{3}, C\right)$.

(vii) When $(x \in A)$ and $(x \notin B)$, then

$$
\begin{gathered}
{\left[\left(F_{1}, G_{1}, A\right) \cap_{E}\left(F_{2}, G_{2}, B\right)\right]^{c}=\left[\left(F_{1}, G_{1}, A\right)\right]^{c},} \\
{\left[\left(F_{1}, G_{1}, A\right)\right]^{c} \cup_{E}\left[\left(F_{2}, G_{2}, B\right)\right]^{c}=\left[\left(F_{1}, G_{1}, A\right)\right]^{c} .}
\end{gathered}
$$

When $(x \notin A)$ and $(x \in B)$, then

$$
\begin{array}{r}
{\left[\left(F_{1}, G_{1}, A\right) \cap_{E}\left(F_{2}, G_{2}, B\right)\right]^{c}=\left[\left(F_{2}, G_{2}, B\right)\right]^{c},} \\
{\left[\left(F_{1}, G_{1}, A\right)\right]^{c} \cup_{E}\left[\left(F_{2}, G_{2}, B\right)\right]^{c}=\left[\left(F_{2}, G_{2}, B\right)\right]^{c} .}
\end{array}
$$

When $x \in A$ and $x \in B$, then the result is a trivial case. Hence, in either case $\left(\left[\left(F_{1}, G_{1}, A\right) \cap_{E}\left(F_{2}, G_{2}, B\right)\right]^{c}=\right.$ $\left.\left[\left(F_{1}, G_{1}, A\right)\right]^{c} \cup_{E}\left[\left(F_{2}, G_{2}, B\right)\right]^{c}\right)$ Similarly, $\left(\left[\left(F_{1}, G_{1}, A\right)\right.\right.$ $\left.\left.\cup_{E}\left(F_{2}, G_{2}, B\right)\right]^{c}=\left[\left(F_{1}, G_{1}, A\right)\right]^{c} \cap_{E}\left[\left(F_{2}, G_{2}, B\right)\right]^{c}\right)$.

Remark 3. For any arbitrary $\left(F_{1}, G_{1}, A\right)\left(F_{2}, G_{2}, B\right)$, $\left(F_{3}, G_{3}, C\right) \in(T-B S S)_{(U)}$, it is not necessary that

(i) $\left(F_{1}, G_{1}, A\right) \cap_{E}\left[\left(F_{2}, G_{2}, B\right) \cup_{E}\left(F_{3}, G_{3}, C\right)\right]=\left[\left(F_{1}\right.\right.$, $\left.\left.G_{1}, A\right) \cap \cap_{E}\left(F_{2}, G_{2}, B\right)\right] \cup_{E}\left[\left(F_{1}, G_{1}, A\right) \cap_{E}\left(F_{3}, G_{3}, C\right)\right]$

(ii) $\left(F_{1}, G_{1}, A\right) \cup_{E}\left[\left(F_{2}, G_{2}, B\right) \cap_{E}\left(F_{3}, G_{3}, C\right)\right]=\left[\left(F_{1}\right.\right.$, $\left.\left.G_{1}, A\right) \cup_{E}\left(F_{2}, G_{2}, B\right)\right] \cap_{E}\left[\left(F_{1}, G_{1}, A\right) \cup_{E}\left(F_{3}, G_{3}, C\right)\right]$

Example 3. Let $E=\left\{e_{1}, e_{2}, e_{3}, e_{4}, e_{5}\right\}, A=\left\{e_{1}, e_{2}, e_{3}\right\}, B=$ $\left\{e_{3}, e_{4}\right\}, \quad C=\left\{e_{4}, e_{5}\right\}, U=\left\{x_{1}, x_{2}, x_{3}, x_{4}, x_{5}\right\}, \quad X=\left\{x_{1}, x_{2}\right.$, $\left.x_{3}\right\}$, and $Y=\left\{x_{4}, x_{5}\right\}$.

Now let

$$
\begin{aligned}
\left(F_{1}, G_{1}, A\right)= & \left\{\left\langle e_{1},\left\{x_{1}, x_{2}\right\},\left\{x_{4}\right\}\right\rangle,\left\langle e_{2},\left\{x_{1}\right\},\left\{x_{4}, x_{5}\right\}\right\rangle,\right. \\
& \left.\cdot\left\langle e_{3},\left\{x_{1}, x_{3}\right\},\left\{x_{4}\right\}\right\rangle\right\}, \\
\left(F_{2}, G_{2}, B\right)= & \left\{\left\langle e_{3},\left\{x_{2}, x_{3}\right\},\left\{x_{5}\right\}\right\rangle,\left\langle e_{4},\left\{x_{1}, x_{2}\right\},\left\{x_{4}, x_{5}\right\}\right\rangle\right\}, \\
\left(F_{3}, G_{3}, C\right)= & \left\{\left\langle e_{4},\left\{x_{1}, x_{2}, x_{3}\right\}, \varnothing\right\rangle,\left\langle e_{5},\left\{x_{1}, x_{2}\right\},\left\{x_{5}\right\}\right\rangle\right\} .
\end{aligned}
$$

Now

$$
\begin{aligned}
\left(F_{1}, G_{1}, A\right) \cap \cap_{E}\left[\left(F_{2}, G_{2}, B\right) \cup_{E}\left(F_{3}, G_{3}, C\right)\right] \\
=\left(F_{1}, G_{1}, A\right) \cap_{E}\left\{\left\langle e_{3},\left\{x_{2}, x_{3}\right\},\left\{x_{5}\right\}\right\rangle,\left\langle e_{4},\left\{x_{1}, x_{2}, x_{3}\right\}, \varnothing\right\rangle,\right. \\
\left.\quad \cdot\left\langle e_{5},\left\{x_{1}, x_{2}\right\},\left\{x_{5}\right\}\right\rangle\right\}, \\
=\left\{\left\langle e_{1},\left\{x_{1}, x_{2}\right\},\left\{x_{4}\right\}\right\rangle,\left\langle e_{2},\left\{x_{1}\right\},\left\{x_{4}, x_{5}\right\}\right\rangle,\right. \\
\left.\left\langle e_{3},\left\{x_{3}\right\},\left\{x_{4}, x_{5}\right\}\right\rangle,\left\langle e_{4},\left\{x_{1}, x_{2}, x_{3}\right\}, \varnothing\right\rangle,\left\langle e_{5},\left\{x_{1}, x_{2}\right\},\left\{x_{5}\right\}\right\rangle\right\} .
\end{aligned}
$$

Next

$$
\begin{aligned}
& \quad\left[\left(F_{1}, G_{1}, A\right) \cap_{E}\left(F_{2}, G_{2}, B\right)\right] \cup_{E}\left[\left(F_{1}, G_{1}, A\right) \cap_{E}\left(F_{3}, G_{3}, C\right)\right]\left\{\left\langle e_{1},\left\{x_{1}, x_{2}\right\},\left\{x_{4}\right\}\right\rangle,\left\langle e_{2},\left\{x_{1}\right\},\left\{x_{4}, x_{5}\right\}\right\rangle,\right. \\
& \left.\quad \cdot\left\langle e_{3},\left\{x_{3}\right\},\left\{x_{4}, x_{5}\right\}\right\rangle,\left\langle e_{4},\left\{x_{1}, x_{2}\right\},\left\{x_{4}, x_{5}\right\}\right\rangle\right\} \cup_{E} \\
& \left\{\left\langle e_{1},\left\{x_{1}, x_{2}\right\},\left\{x_{4}\right\}\right\rangle,\left\langle e_{2},\left\{x_{1}\right\},\left\{x_{4}, x_{5}\right\}\right\rangle,\left\langle e_{3},\left\{x_{1}, x_{3}\right\},\left\{x_{4}\right\}\right\rangle,\left\langle e_{4},\left\{x_{1}, x_{2}, x_{3}\right\}, \varnothing\right\rangle,\left\langle e_{5},\left\{x_{1}, x_{2}\right\},\left\{x_{5}\right\}\right\rangle\right\} \\
& =\left\{\left\langle e_{1},\left\{x_{1}, x_{2}\right\},\left\{x_{4}\right\}\right\rangle,\left\langle e_{2},\left\{x_{1}\right\},\left\{x_{4}, x_{5}\right\}\right\rangle,\left\langle e_{3},\left\{x_{1}, x_{3}\right\},\left\{x_{4}\right\}\right\rangle,\left\langle e_{4},\left\{x_{1}, x_{2}, x_{3}\right\}, \varnothing\right\rangle,\left\langle e_{5},\left\{x_{1}, x_{2}\right\},\left\{x_{5}\right\}\right\rangle\right\}, \\
& \Longrightarrow\left(F_{1}, G_{1}, A\right) \cap_{E}\left[\left(F_{2}, G_{2}, B\right) \cup \cup_{E}\left(F_{3}, G_{3}, C\right)\right] \neq\left[\left(F_{1}, G_{1}, A\right) \cap_{E}\left(F_{2}, G_{2}, B\right)\right] \cup \cup_{E}\left[\left(F_{1}, G_{1}, A\right) \cap E\left(F_{3}, G_{3}, C\right)\right] .
\end{aligned}
$$


Now

$$
\begin{gathered}
\left(F_{1}, G_{1}, A\right) \cup_{E}\left[\left(F_{2}, G_{2}, B\right) \cap \cap_{E}\left(F_{3}, G_{3}, C\right)\right]=\left(F_{1}, G_{1}, A\right) \cup_{E}\left\{\left\langle e_{3},\left\{x_{2}, x_{3}\right\},\left\{x_{5}\right\}\right\rangle,\left\langle e_{4},\left\{x_{1}, x_{2}\right\},\left\{x_{4}, x_{5}\right\}\right\rangle,\left\langle e_{5},\left\{x_{1}, x_{2}\right\},\left\{x_{5}\right\}\right\rangle\right\} \\
\quad=\left\{\left\langle e_{1},\left\{x_{1}, x_{2}\right\},\left\{x_{4}\right\}\right\rangle,\left\langle e_{2},\left\{x_{1}\right\},\left\{x_{4}, x_{5}\right\}\right\rangle,\left\langle e_{3},\left\{x_{1}, x_{2}, x_{3}\right\}, \varnothing\right\rangle,\left\langle e_{4},\left\{x_{1}, x_{2}\right\},\left\{x_{4}, x_{5}\right\}\right\rangle,\left\langle e_{5},\left\{x_{1}, x_{2}\right\},\left\{x_{5}\right\}\right\rangle\right\} .
\end{gathered}
$$

Next

$$
\begin{aligned}
& {\left[\left(F_{1}, G_{1}, A\right) \cup_{E}\left(F_{2}, G_{2}, B\right)\right] \cap_{E}\left[\left(F_{1}, G_{1}, A\right) \cup \cup_{E}\left(F_{3}, G_{3}, C\right)\right]=\left\{\left\langle e_{1},\left\{x_{1}, x_{2}\right\},\left\{x_{4}\right\}\right\rangle,\left\langle e_{2},\left\{x_{1}\right\},\left\{x_{4}, x_{5}\right\}\right\rangle\right.} \\
& \left.\quad \cdot\left\langle e_{3},\left\{x_{1}, x_{2}, x_{3}\right\}, \varnothing\right\rangle,\left\langle e_{4},\left\{x_{1}, x_{2}\right\},\left\{x_{4}, x_{5}\right\}\right\rangle\right\} \cap E \\
& \left\{\left\langle e_{1},\left\{x_{1}, x_{2}\right\},\left\{x_{4}\right\}\right\rangle,\left\langle e_{2},\left\{x_{1}\right\},\left\{x_{4}, x_{5}\right\}\right\rangle,\left\langle e_{3},\left\{x_{1}, x_{3}\right\},\left\{x_{4}\right\}\right\rangle,\left\langle e_{4},\left\{x_{1}, x_{2}, x_{3}\right\}, \varnothing\right\rangle,\left\langle e_{5},\left\{x_{1}, x_{2}\right\},\left\{x_{5}\right\}\right\rangle\right\} \\
& =\left\{\left\langle e_{1},\left\{x_{1}, x_{2}\right\},\left\{x_{4}\right\}\right\rangle,\left\langle e_{2},\left\{x_{1}\right\},\left\{x_{4}, x_{5}\right\}\right\rangle,\left\langle e_{3},\left\{x_{1}, x_{3}\right\},\left\{x_{4}\right\}\right\rangle,\left\langle e_{4},\left\{x_{1}, x_{2}\right\},\left\{x_{4}, x_{5}\right\}\right\rangle,\left\langle e_{5},\left\{x_{1}, x_{2}\right\},\left\{x_{5}\right\}\right\rangle\right\} \\
& \Longrightarrow\left(F_{1}, G_{1}, A\right) \cup_{E}\left[\left(F_{2}, G_{2}, B\right) \cap \cap_{E}\left(F_{3}, G_{3}, C\right)\right] \neq\left[\left(F_{1}, G_{1}, A\right) \cup \cup_{E}\left(F_{2}, G_{2}, B\right)\right] \cap \cap_{E}\left[\left(F_{1}, G_{1}, A\right) \cup \cup_{E}\left(F_{3}, G_{3}, C\right)\right] .
\end{aligned}
$$

Definition 20. Let $\left(F_{1}, G_{1}, A\right) \quad\left(F_{2}, G_{2}, B\right) \in(T-B S S)_{(U)}$ with $A \cap B \neq \varnothing$. Then, (i) "Restricted union" of $\left(F_{1}, G_{1}, A\right)$ and $\left(F_{2}, G_{2}, B\right)$ is designated and demarcated by

$$
\left(F_{1}, G_{1}, A\right) \cup_{R}\left(F_{2}, G_{2}, B\right)=\left\{\left\langle c, F_{1}(c) \cup F_{2}(c), G_{1}(c) \cap G_{2}(c)\right\rangle: c \in A \cap B\right\}
$$

(ii) "Restricted intersection" of $\left(F_{1}, G_{1}, A\right)$ and $\left(F_{2}, G_{2}, B\right)$ is designated and demarcated by

$$
\left(F_{1}, G_{1}, A\right) \cap_{R}\left(F_{2}, G_{2}, B\right)=\left\{\left\langle c, F_{1}(c) \cap F_{2}(c), G_{1}(c) \cup G_{2}(c)\right\rangle: c \in A \cap B\right\}
$$

Proposition 3. For any T-BSSs $\left(F_{1}, G_{1}, A\right),\left(F_{2}, G_{2}, B\right)$, and $\left(F_{3}, G_{3}, C\right)$,

(i) $\left(F_{1}, G_{1}, A\right) \cap_{R} \phi=\phi,\left(F_{1}, G_{1}, A\right) \cup_{R} \phi=\left(F_{1}\right.$, $\left.G_{1}, A\right),\left(F_{1}, G_{1}, A\right) \cap \cap_{R} \mathscr{A}=\left(F_{1}, G_{1}, A\right),\left(F_{1}, G_{1}\right.$, A) $\cup_{R} \mathscr{A}=\mathscr{A}$

(ii) $\left(F_{1}, G_{1}, A\right) \cap_{R}\left(F_{1}, G_{1}, A\right)=\left(F_{1}, G_{1}, A\right),\left(F_{1}, G_{1}\right.$, A) $\cup_{R}\left(F_{1}, G_{1}, A\right)=\left(F_{1}, G_{1}, A\right)$

(iii) $\left(F_{1}, G_{1}, A\right) \cap_{R}\left(F_{2}, G_{2}, B\right)=\left(F_{2}, G_{2}, B\right) \cap_{R}\left(F_{1}, G_{1}\right.$, $A),\left(F_{1}, G_{1}, A\right) \cup_{R}\left(F_{2}, G_{2}, B\right)=\left(F_{2}, G_{2}, B\right) \cup_{R}\left(F_{1}\right.$, $\left.G_{1}, A\right),\left(F_{1}, G_{1}, A\right) \cup_{R}\left(F_{2}, G_{2}, B\right)=\left(F_{2}, G_{2}, B\right) \cup_{R}$ $\left(F_{1}, G_{1}, A\right), \quad\left(F_{1}, G_{1}, A\right) \cup{ }_{R}\left(F_{2}, G_{2}, B\right)=\left(F_{2}, G_{2}\right.$, B) $\cup_{R}\left(F_{1}, G_{1}, A\right)$

(iv) $\left(F_{1}, G_{1}, A\right) \cap_{R}\left[\left(F_{2}, G_{2}, B\right) \cap_{R}\left(F_{3}, G_{3}, C\right)\right]=\left[\left(F_{1}\right.\right.$, $\left.\left.G_{1}, A\right) \cap_{R}\left(F_{2}, G_{2}, B\right)\right] \cap_{R}\left(F_{3}, G_{3}, C\right), \quad\left(F_{1}, G_{1}, A\right)$
$\cup_{R}\left[\left(F_{2}, G_{2}, B\right) \cup_{R}\left(F_{3}, G_{3}, C\right)\right]=\left[\left(F_{1}, G_{1}, A\right) \cup_{R}\right.$ $\left.\left(F_{2}, G_{2}, B\right)\right] \cup_{R}\left(F_{3}, G_{3}, C\right)$

(v) $\left(F_{1}, G_{1}, A\right) \cap{ }_{R}\left[\left(F_{2}, G_{2}, B\right) \cup{ }_{R}\left(F_{3}, G_{3}, C\right)\right]=\left[\left(F_{1}\right.\right.$, $\left.\left.G_{1}, A\right) \cap{ }_{R}\left(F_{2}, G_{2}, B\right)\right] \cup_{R}\left[\left(F_{1}, G_{1}, A\right) \cap_{R}\left(F_{3}, G_{3}\right.\right.$, $C)], \quad\left(F_{1}, G_{1}, A\right) \cup_{R}\left[\left(F_{2}, G_{2}, B\right) \cap_{R}\left(F_{3}, G_{3}, C\right)\right]=$ $\left[\left(F_{1}, G_{1}, A\right) \cup_{R}\left(F_{2}, G_{2}, B\right)\right] \cap_{R}\left[\left(F_{1}, G_{1}, A\right) \cup_{R}\left(F_{3}\right.\right.$, $\left.\left.G_{3}, C\right)\right]$

(vi) $\left(F_{1}, G_{1}, A\right) \cap_{R}\left[\left(F_{2}, G_{2}, B\right) \cup_{R}\left(F_{1}, G_{1}, A\right)\right]=\left(F_{1}\right.$, $\left.G_{1}, A\right),\left(F_{1}, G_{1}, A\right) \cup_{R}\left[\left(F_{2}, G_{2}, B\right) \cap_{R}\left(F_{1}, G_{1}, A\right)\right]=$ $\left(F_{1}, G_{1}, A\right)$

(vii) $\left[\left(F_{1}, G_{1}, A\right)^{c}\right]^{c}=\left(F_{1}, G_{1}, A\right),\left(F_{1}, G_{1}, A\right) \cap_{R}\left[\left(F_{1}\right.\right.$, $\left.\left.G_{1}, A\right)\right]^{c}=\phi,\left(F_{1}, G_{1}, A\right) \cup_{R}\left[\left(F_{1}, G_{1}, A\right)\right]^{c}=\mathscr{A}$

(viii) $\left[\left(F_{1}, G_{1}, A\right) \cap{ }_{R}\left(F_{2}, G_{2}, B\right)\right]^{c}=\left[\left(F_{1}, G_{1}, A\right)\right]^{c} \cup_{R}$ $\left[\left(F_{2}, G_{2}, B\right)\right]^{c},\left[\left(F_{1}, G_{1}, A\right) \cup{ }_{R}\left(F_{2}, G_{2}, B\right)\right]^{c}=\left[\left(F_{1}\right.\right.$, $\left.\left.G_{1}, A\right)\right]^{c} \cap_{R}\left[\left(F_{2}, G_{2}, B\right)\right]^{c}$ 
Proof. Straightforward.

\section{Algebraic Structures Associated with T-BSSs}

In this section, we will discuss some algebraic structures associated with T-BSSs. Recall that $(\mathrm{T}-\mathrm{BSS})_{(U)}$ denotes the collection of all T-BSSs over $U$. Now in this section, $(\mathrm{T}-\mathrm{BSS})_{(U)}^{A}$ denotes the collection of all T-BSSs over $U$ with domain $A$.

Proposition 4. For any $\Delta \in\left\{\cap_{E}, \cup_{E}, \cap_{R}, \cup_{R}\right\}$, $\left((T-B S S)_{(U)}, \Delta\right)$ is a commutative semigroup whose every element is idempotent.

Proof. The proof is straightforward by using the Proposition 2 and Proposition 3.

Proposition 5. $\left((T-B S S)_{(U)}, \cap_{R}, \cup_{R}\right)$ is a commutative semiring.

Proof. The proof follows from the definitions of restricted intersection of T-BSSs, restricted union of T-BSSs, and parts (iv) and (v) of Proposition 3.

Remark 4. It follows from Proposition 2 and Remark 3 that $\left((\mathrm{T}-\mathrm{BSS})_{(U)}, \cap_{E}, \cup_{E}\right)$ is not a semiring.

Proposition 6. $\left((T-B S S)_{(U)}^{A}, \cap_{E}, \cup_{E}\right)$ is a commutative semiring.

Proof. This is straightforward as extended intersection of T-BSSs and extended union of T-BSSs satisfy the distributive laws, which usually do not hold (Remark 3 ), if all the T-BSSs have same domain A.

Proposition 7. $(T-B S S)_{(U)}, \cap_{E}, \cup_{E},{ }^{c},(\phi, \mathscr{A})$ is a bounded lattice.

Proof. The result follows from conditions (i)-(v) of Proposition 2 .

Proposition 8. $(T-B S S)_{(U)}, \cap_{R}, \cup_{R},{ }^{c},(\phi, \mathscr{A})$ is a bounded distributive lattice.

Proof. The result follows from Proposition 3.

\section{Applications of T-BSSs in Decision Making}

In this section, we will discuss some decision-making problems by using T-BSSs. We will discuss decision-making problems in the absence of weights, in the presence of weights selected randomly, and in the presence of weights taken as discussed in [48].

Definition 21. Let $A=\left\{a_{1}, a_{2}, a_{3}, \ldots, a_{l}\right\} \subseteq E, X=\left\{x_{1}, x_{2}\right.$, $\left.x_{3}, \ldots, x_{m}\right\}, Y=\left\{y_{1}, y_{2}, y_{3}, \ldots, y_{n}\right\}$, and $(F, G, A)$ be corresponding T-BSS. Then, score of $\left(a_{i}, 1 \leq i \leq l\right)$ is denoted and defined as $\left(S_{i}=\overline{\sigma_{i}}-\sigma_{i}\right)$, where $\left(\overline{\sigma_{i}}=\sum_{j, k} \zeta_{\mathrm{ijk}}^{*}\right)$ and $\left(\sigma_{i}=\sum_{j, k} \zeta_{\mathrm{ijk}}^{0}\right)$.
Definition 22. Let $A=\left\{a_{1}, a_{2}, a_{3}, \ldots, a_{l}\right\} \subseteq E, X=\left\{x_{1}, x_{2}\right.$, $\left.x_{3}, \ldots, x_{m}\right\}, Y=\left\{y_{1}, y_{2}, y_{3}, \ldots, y_{n}\right\}$, and $(F, G, A)$ be corresponding T-BSS. Then, $\left(a_{i}, 1 \leq i \leq l\right)$ is said to be optimal if and only if $\left(S_{i}>S_{i^{\prime}}\right)$, for all $\left(i^{\prime} \neq i\right)$.

Example 4. Consider Example 2 with $(F, G, A)=\left\{\left\langle a_{1}\right.\right.$, $\left.\left\{x_{1}, x_{3}\right\},\left\{y_{3}\right\}\right\rangle,\left\langle a_{2},\left\{x_{1}, x_{2}, x_{4}\right\},\left\{y_{1}\right\}\right\rangle,\left\langle a_{3},\left\{x_{1}, \quad x_{4}\right\},\left\{y_{1}\right.\right.$, $\left.\left.\left.y_{3}\right\}\right\rangle,\left\langle a_{4},\left\{x_{2}, x_{3}, x_{4}\right\},\left\{y_{2}, y_{3}\right\}\right\rangle,\left\langle a_{5},\left\{x_{2}, x_{4}\right\},\left\{y_{2}\right\}\right\rangle\right\}$, with tabular form as in Table 3.

Then, the score values are given in Table 4.

Then, according to the Algorithm 1 , the candidate " $a_{2}$ " will be selected.

Remark 5. Sometimes in decision making, some decision makers have less importance as compared to other decision makers, for example, to decide about admission policy of a school, a meeting was called in which four persons participated who were the school owner, school principal, school vice principal, and accountant of the school. Now here it is clear that all the decision makers have not the same weightage. So, in decision making, the weightage of a decision maker also matters a lot. So, now we establish an algorithm to handle a decision-making problem in the presence of weights.

Definition 23. Let $A=\left\{a_{1}, a_{2}, a_{3}, \ldots, a_{l}\right\} \subseteq E, X=\left\{x_{1}, x_{2}\right.$, $\left.x_{3}, \ldots, x_{m}\right\}$, and $Y=\left\{y_{1}, y_{2}, y_{3}, \ldots, y_{n}\right\}$ such that each $x_{j}$ has weight $w_{j}$ and each $y_{k}$ has weight $w_{k}^{\prime}$ with $\sum_{j} w_{j}=1 \& \sum_{k} w_{k}^{\dagger}=1$. Then, for all $i,\left(S_{i}=\overline{\sigma_{i}}-\sigma_{i}\right)$, where $\overline{\sigma_{i}}=\sum_{j, k} w_{j} \zeta_{i j k}^{*}$ and $\sigma_{i}=\sum_{j, k} w_{k}^{\prime} \zeta_{i j k}^{\circ}$.

Remark 6. The above stated algorithm (Algorithm 1) also works in the present case.

Example 5. Consider Example 4, with Table 5 representing weight values and Table 6 representing score values.

Then, according to the new criteria, the candidate " $a_{1}$ " will be selected.

Remark 7. According to $\mathrm{Xu}$ [48], the weight vector $w=$ $\left(w_{1}, w_{2}, w_{3}, \ldots, w_{n}\right)^{T}$ can also be calculated as

$$
w_{i}=\frac{e^{-\left[\left(\left(j-\mu_{n}\right)^{2} / 2 \sigma_{n}^{2}\right)\right]}}{\sum_{j=1}^{n} e^{-\left[\left(\left(j-\mu_{n}\right)^{2} / 2 \sigma_{n}^{2}\right)\right]}},
$$

where

$$
\begin{aligned}
\mu_{n} & =\frac{1+n}{2}, \\
\sigma_{n} & =\sqrt{\frac{1}{n} \sum_{i=1}^{n}\left(i-\mu_{n}\right)^{2} .}
\end{aligned}
$$

In this case, Example 5 takes the following form (Tables 7 and 8 ).

Then, in this case, the candidate " $a_{2}$ " will be selected. 
TABLE 3: Tabular expression of the T-bipolar soft set $(F, G, A)$.

\begin{tabular}{lccccccccccccc}
\hline$(F, G, A)$ & $\left(x_{1}, y_{1}\right)$ & $\left(x_{1}, y_{2}\right)$ & $\left(x_{1}, y_{3}\right)$ & $\left(x_{2}, y_{1}\right)$ & $\left(x_{2}, y_{2}\right)$ & $\left(x_{2}, y_{3}\right)$ & $\left(x_{3}, y_{1}\right)$ & $\left(x_{3}, y_{2}\right)$ & $\left(x_{3}, y_{3}\right)$ & $\left(x_{4}, y_{1}\right)$ & $\left(x_{4}, y_{2}\right)$ & $\left(x_{4}, y_{3}\right)$ \\
\hline$a_{1}$ & $(1,0)$ & $(1,0)$ & $(1,1)$ & $(0,0)$ & $(0,0)$ & $(0,1)$ & $(1,0)$ & $(1,0)$ & $(1,1)$ & $(0,0)$ & $(0,0)$ & $(0,1)$ \\
$a_{2}$ & $(1,1)$ & $(1,0)$ & $(1,0)$ & $(1,1)$ & $(1,0)$ & $(1,0)$ & $(0,1)$ & $(0,0)$ & $(0,0)$ & $(1,1)$ & $(1,0)$ & $(1,0)$ \\
$a_{3}$ & $(1,1)$ & $(1,0)$ & $(1,1)$ & $(0,1)$ & $(0,0)$ & $(0,1)$ & $(0,1)$ & $(0,0)$ & $(0,1)$ & $(1,1)$ & $(1,0)$ & $(1,1)$ \\
$a_{4}$ & $(0,0)$ & $(0,1)$ & $(0,1)$ & $(1,0)$ & $(1,1)$ & $(1,1)$ & $(1,0)$ & $(1,1)$ & $(1,1)$ & $(1,0)$ & $(1,1)$ & $(1,1)$ \\
$a_{5}$ & $(0,0)$ & $(0,1)$ & $(0,0)$ & $(1,0)$ & $(1,1)$ & $(1,0)$ & $(0,0)$ & $(0,1)$ & $(0,0)$ & $(1,0)$ & $(1,1)$ & $(1,0)$ \\
\hline
\end{tabular}

TABLE 4: Scores of $a_{1}, a_{2}, a_{3}, a_{4}, a_{5}$.

\begin{tabular}{lccc}
\hline$(F, G, A)$ & $\overline{\sigma_{i}}$ & $\underline{\sigma_{i}}$ & \\
\hline$a_{1}$ & 6 & 4 & $S_{i}$ \\
$a_{2}$ & 9 & 4 & 2 \\
$a_{3}$ & 6 & 8 & 5 \\
$a_{4}$ & 9 & 8 & -2 \\
$a_{5}$ & 6 & 4 & 1 \\
\hline
\end{tabular}

(1 Here we state an algorithm for finding an optimal value for a given data. Step 1. Write given T-BSS in tabular form.

Step 2. Calculate $S_{1}, S_{2}, S_{3}, \ldots, S_{l}$.

Step 3. Put $\max S_{i}=S_{p}$.

Step 4. $S_{p}$ is optimal value.

Algorithm 1: Finding an optimal value for a given data.

TABLE 5: Weight values.

\begin{tabular}{cccc}
\hline$x_{j}$ & Weight of $x_{j}$ & $y_{k}$ & Weight of $y_{k}$ \\
\hline$x_{1}$ & 0.3 & $y_{1}$ & 0.4 \\
$x_{2}$ & 0.2 & $y_{2}$ & 0.4 \\
$x_{3}$ & 0.4 & $y_{3}$ & 0.2 \\
$x_{4}$ & 0.1 & - & - \\
\hline
\end{tabular}

TABLE 6: Scores of $a_{1}, a_{2}, a_{3}, a_{4}, a_{5}$.

\begin{tabular}{llll}
\hline$(F, G, A)$ & $\overline{\sigma_{i}}$ & $\underline{\sigma_{i}}$ & $S_{i}$ \\
\hline$a_{1}$ & 2.1 & 0.8 & 1.3 \\
$a_{2}$ & 1.8 & 1.6 & 0.2 \\
$a_{3}$ & 1.2 & 2.4 & -1.2 \\
$a_{4}$ & 2.1 & 2.4 & -0.3 \\
$a_{5}$ & 0.9 & 1.6 & -0.7 \\
\hline
\end{tabular}

TABLE 7: Weight values.

\begin{tabular}{cccc}
\hline$x_{j}$ & Weight of $x_{j}$ & $y_{k}$ & Weight of $y_{k}$ \\
\hline$x_{1}$ & 0.1550 & $y_{1}$ & 0.2429 \\
$x_{2}$ & 0.3450 & $y_{2}$ & 0.5142 \\
$x_{3}$ & 0.3450 & $y_{3}$ & 0.2429 \\
$x_{4}$ & 0.1550 & - & - \\
\hline
\end{tabular}

TABLE 8: Scores of $a_{1}, a_{2}, a_{3}, a_{4}, a_{5}$.

\begin{tabular}{lccc}
\hline$(F, G, A)$ & $\overline{\sigma_{i}}$ & $\underline{\sigma_{i}}$ & $S_{i}$ \\
\hline$a_{1}$ & 1.5 & 0.9716 & 0.5284 \\
$a_{2}$ & 1.965 & 0.9716 & 0.9934 \\
$a_{3}$ & 0.93 & 1.9432 & -1.0132 \\
$a_{4}$ & 2.535 & 3.0284 & -0.4934 \\
$a_{5}$ & 1.5 & 2.0568 & -0.5568 \\
\hline
\end{tabular}

\section{Conclusion and Future Prospective}

Keeping in view the shortcoming in predefined notions of BSSs, in this article, we have defined and discussed the notion of T-BSS. Then, rendering to new definition, we have defined different binary operations for T-BSSs and then we conferred some results associated with these binary operations. We evidenced the existence of bounded lattices and De Morgan 
algebras interrelated with these binary operations. We also established some algorithms to solve decision-making problems and then solved the problems from daily life by using these algorithms. In future, this work can be extended to its applications in algebraic structures and in rough set theory.

\section{Data Availability}

The data used in this article are artificial and hypothetical, and anyone can use these data before prior permission by just citing this article.

\section{Conflicts of Interest}

The author declares that there are no conflicts of interest.

\section{References}

[1] L. A. Zadeh, "Fuzzy sets," Information and Control, vol. 8, no. 3, pp. 338-353, 1965.

[2] Z. Pawlak, "Rough sets," International Journal of Computing and Information Science, vol. 11, pp. 341-356, 1982.

[3] D. A. Molodtsov, "Soft set theory-first results," Computers and Mathematics with Applications, vol. 37, no. 4-5, pp. 19-31, 1999.

[4] P. K. Maji, R. Bismas, and A. R. Roy, "Soft set theory," Computers and Mathematics with Applications, vol. 45, no. 45, pp. 555-562, 2003.

[5] M. I. Ali, F. Feng, X. Y. Liu, W. K. Min, and M. Shabir, "On some new operations in soft set theory," Computers and Mathematics with Applications, vol. 57, no. 9, pp. 1547-1553, 2009.

[6] M. I. Ali, M. Shabir, and M. Naz, "Algebraic structures of soft sets associated with new operations," Computers and Mathematics with Applications, vol. 61, no. 9, pp. 2647-2654, 2011.

[7] H. Aktaş and N. Çağman, "Soft sets and soft groups," Information Sciences, vol. 177, no. 13, pp. 2726-2735, 2007.

[8] U. Acar, F. Koyuncu, and B. Tanay, "Soft sets and soft rings," Computers and Mathematics with Applications, vol. 59, no. 11, pp. 3458-3463, 2010.

[9] A. S. Sezer and A. O. Atagün, "A new kind of vector space: soft vector space," Southeast Asian Bulletin of Mathematics, vol. 40, no. 5, pp. 753-770, 2016.

[10] M. I. Ali, M. Shabir, and F. Feng, "Representation of graphs based on neighborhoods and soft sets," International Journal of Machine Learning and Cybernetics, vol. 8, no. 5, pp. 1525-1535, 2017.

[11] M. Shabir and M. Naz, "On soft topological spaces," Computers and Mathematics with Applications, vol. 61, no. 7, pp. 1786-1799, 2011.

[12] A. S. Sezer, N. Çağman, A. O. Atagün, M. I. Ali, and E. Türkmen, "Soft intersection semigroups, ideals and biideals; a new application on semigroup theory I," Filomat, vol. 29, no. 5, pp. 917-946, 2015.

[13] M. I. Ali, T. Mahmood, M. M. U. Rehman, and M. F. Aslam, "On lattice ordered soft sets," Applied Soft Computing, vol. 36, pp. 499-505, 2015.

[14] N. Cagman, "Contributions to the theory of soft sets," Journal of New Results in Science, vol. 4, pp. 33-41, 2014.

[15] P. K. Maji, R. Biswas, and R. Roy, "An application of soft sets in decision-making problems," Computers and Mathematics with Applications, vol. 44, pp. 1077-1083, 2002.
[16] N. Cagman and S. Enginoglu, "Soft set theory and uni-int decision making," European Journal of Operational Research, vol. 207 , no. 2, pp. 848-855, 2010.

[17] N. Cagman and S. Enginoglu, "Soft matrices and its decision makings," Computers and Mathematics with Applications, vol. 59, pp. 3308-3314, 2010.

[18] Z. Kong, G. Zhang, L. Wang, Z. Wu, S. Qi, and H. Wang, "An efficient decision making approach in incomplete soft set," Applied Mathematical Modelling, vol. 38, no. 7-8, pp. 21412150, 2014.

[19] P. Zhu and Q. Wen, "Operations on soft sets revisited," Journal of Mathematics, vol. 2013, no. 7, Article ID 105752, 2013.

[20] M. Zhou, S. Li, and M. Akram, "Categorical properties of soft sets," The Scientific World Journal, vol. 2014, no. 10, Article ID 783056, 2014.

[21] M. I. Siddique, T. Mahmood, and N. Jan, "On double framed soft rings," Technical Journal UET Taxila, vol. 25, no. 2, pp. 126-131, 2020.

[22] T. Mahmood, Z. U. Rehman, and A. S. Sezer, "Lattice ordered soft near rings," Korean Journal of Mathematics, vol. 26, no. 3, pp. 503-517, 2018.

[23] M. Iftikhar and T. Mahmood, "Some results on lattice ordered double framed soft semirings," International Journal of Algebra and Statistics, vol. 7, no. 1-2, pp. 123-140, 2018.

[24] P. K. Maji, R. Biswas, and A. R. Roy, "Fuzzy soft sets," Journal of Fuzzy Mathematics, vol. 9, no. 3, pp. 589-602, 2001.

[25] T. Deng and X. Wang, "An object-parameter approach to predicting unknown data in incomplete fuzzy soft sets," Applied Mathematical Modelling, vol. 37, no. 6, pp. 41394146, 2013.

[26] M. Naz and M. Shabir, "Fuzzy soft sets, and their algebraic structures," World Applied Sciences Journal (Special Issue of Applied Math), vol. 22, pp. 45-61, 2013.

[27] A. R. Roy and P. K. Maji, "A fuzzy soft set theoretic approach to decision-making problems," Journal of Computational and Applied Mathematics, vol. 203, no. 2, pp. 412-418, 2007.

[28] N. Cagman, S. Enginoglu, and F. Erdogan, "Fuzzy soft set theory and its applications," Iranian Journal of Fuzzy Systems, vol. 8, no. 3, pp. 137-147, 2011.

[29] F. Feng, Y. B. Jun, X. Liu, and L. Li, “An adjustable approach to fuzzy soft sets based decision making," Journal of Computational and Applied Mathematics, vol. 234, pp. 10-20, 2010.

[30] F. Feng, C. Li, B. Davvaz, and M. I. Ali, "Soft sets combined with fuzzy sets and rough sets: a tentative approach," Soft Computing, vol. 14, no. 9, pp. 899-911, 2010.

[31] T. Som, "On the theory of soft sets, soft relation and fuzzy soft relation," in Proceedings of the National Conference on Uncertainty: A Mathematical Approach, UAMA-06, pp. 1-9, Burdwan, India, 2006.

[32] D. K. Sut, "An application of fuzzy soft relation in decision making problems," International Journal of Mathematics Trends and Technology, vol. 3, no. 2, pp. 51-54, 2012.

[33] Z. Xiao, K. Gong, and Y. Zou, "A combined forecasting approach based on fuzzy soft sets," Journal of Computational and Applied Mathematics, vol. 228, no. 1, pp. 326-333, 2009.

[34] M. F. Aslam, M. I. Ali, T. Mahmood, M. M. U. Rehman, and N. Sarfraz, "Study of fuzzy soft sets with some order on set of parameters," International Journal of Algebra and Statistics, vol. 8, pp. 50-65, 2019.

[35] K. M. Lee, "Bipolar valued fuzzy sets and their operations," in Proceedings of the International Conference on Intelligent 
Technologies, pp. 307-312, Bangkok, Thailand, December 2000.

[36] S. Abdullah, M. Aslam, and K. Ullah, "Bipolar fuzzy soft sets and its applications in decision making problem," Journal of Intelligent \& Fuzzy Systems, vol. 27, no. 2, pp. 729-742, 2014.

[37] M. Shabir and M. Naz, "On bipolar soft sets," 2013, https:// arxiv.org/abs/1303.1344.

[38] M. Naz and M. Shabir, "On fuzzy bipolar soft sets, their algebraic structures and applications," Journal of Intelligent and Fuzzy Systems, vol. 26, no. 4, pp. 1645-1656, 2014.

[39] F. Karaaslan and S. Karatas, "A new approach to bipolar soft sets and its applications," Discrete Mathematic, Algorithms and Applications, vol. 7, no. 4, Article ID 1550054, 2015.

[40] F. Karaaslan, I. Ahmad, and A. Ullah, "Bipolar soft groups," Journal of Intelligent and Fuzzy Systems, vol. 31, no. 1, pp. 651-662, 2016.

[41] A. Khan, F. Hussain, A. Hadi, and S. A. Khan, "A decision making approach based on multi-fuzzy bipolar soft sets," Journal of Intelligent and Fuzzy Systems, vol. 37, no. 2, pp. 1879-1892, 2019.

[42] A. Khan, M. Izhar, and M. M. Khalaf, "Generalized multifuzzy bipolar soft sets and its applications in decision making," Journal of Intelligent and Fuzzy Systems, vol. 37, no. 2, pp. 2713-2725, 2019.

[43] N. Malik and M. Shabir, "Rough fuzzy bipolar soft sets and application in decision-making Problems," Soft Computing, vol. 23 , no. 5, pp. 1603-1614, 2019.

[44] M. Akram and G. Ali, "Hybrid models for decision-making based on rough Pythagorean fuzzy bipolar soft information," Granular Computing, vol. 5, no. 1, pp. 1-15, 2020.

[45] G. Ali, M. Akram, A. N. A. Koam, and J. C. R. Alcantud, "Parameter reductions of bipolar fuzzy soft sets with their decision-making algorithms," Symmetry, vol. 11, no. 8, p. 949, 2019.

[46] K. Atanassov, "Intuitionistic fuzzy sets," Fuzzy Sets and Systems, vol. 20, no. 1, pp. 87-96, 1986.

[47] Y. B. Jun and S. S. Ahn, "Double-framed soft sets with applications in BCK/BCI-algebras," Journal of Applied Mathematics, vol. 2012, Article ID 178159, 15 pages, 2012.

[48] Z. Xu, "An overview of methods for determining OWA weights," International Journal of Intelligent Systems, vol. 20, no. 8, pp. 843-865, 2005. 